\title{
What makes a pattern? Matching decoding methods to data in multivariate pattern analysis
}

\author{
Philip A. Kragel 1,2, R. McKell Carter ${ }^{2,3}$ and Scott A. Huettel ${ }^{1,2,3 *}$ \\ 1 Department of Psychology and Neuroscience, Duke University, Durham, NC, USA \\ ${ }^{2}$ Center for Cognitive Neuroscience, Duke University, Durham, NC, USA \\ ${ }^{3}$ Brain Imaging and Analysis Center, Duke University, Durham, NC, USA
}

Edited by:

Russell A. Poldrack, University of

Texas, USA

\section{Reviewed by:}

Michael Hanke,

Otto-von-Guericke-University,

Germany

Jeanette Mumford, University of

Texas at Austin, USA

*Correspondence:

Scott A. Huettel, Center for Cognitive Neuroscience, B203 LSRC Building,

Research Drive, Box 90999, Durham NC 27708-0999, USA.

e-mail: scott.huettel@duke.edu
Research in neuroscience faces the challenge of integrating information across different spatial scales of brain function. A promising technique for harnessing information at a range of spatial scales is multivariate pattern analysis (MVPA) of functional magnetic resonance imaging (fMRI) data. While the prevalence of MVPA has increased dramatically in recent years, its typical implementations for classification of mental states utilize only a subset of the information encoded in local fMRI signals. We review published studies employing multivariate pattern classification since the technique's introduction, which reveal an extensive focus on the improved detection power that linear classifiers provide over traditional analysis techniques. We demonstrate using simulations and a searchlight approach, however, that non-linear classifiers are capable of extracting distinct information about interactions within a local region. We conclude that for spatially localized analyses, such as searchlight and region of interest, multiple classification approaches should be compared in order to match $\mathrm{fMRI}$ analyses to the properties of local circuits.

\section{INTRODUCTION}

Our ability to understand brain function is limited by the scale and accuracy with which we can quantify neural activity. Knowledge about brain function comes from different research methods, depending on the spatial scale: individual neurons (Koch, 1997; Dayan and Abbott, 2001; Gerstner and Kistler, 2002), cortical columns, larger brain regions (Rolls and Treves, 1998; Frackowiak, 2004), and functional networks spanning the whole brain (Fox et al., 2005). As one example, recording from single neurons provides exquisitely detailed temporal records of action potentials (up to the millisecond scale) but limits our coverage of the brain to a small volume surrounding the electrode a cross-section of about three square micrometers (Boulton et al., 1990). Conversely, functional magnetic resonance imaging (fMRI) provides information about neural metabolic changes, not neuronal activity, but allows an unparalleled combination of spatial resolution (down to sub-millimeter resolution) and whole brain coverage (Huettel et al., 2008). In spite of this, the spatial resolution of fMRI in typical experiments is still inadequate to describe even columnar structures (Mountcastle, 1997). Gaps between levels of description present barriers both to integrated basic science research and to a more refined treatment of mental disorders.

Recent technological, methodological, and analytical innovations have promised to bridge knowledge across levels of spatial resolution (Kim et al., 2000; Logothetis et al., 2001; Kamitani and Tong, 2005; Kriegeskorte and Bandettini, 2007; Kriegeskorte et al., 2008). Here we inspect the recent introduction of statistical learning techniques to fMRI, often grouped under the term multivariate pattern analysis (MVPA; Haynes and Rees, 2006; Norman et al., 2006; Pereira et al., 2009; Weil and Rees, 2010). MVPA simultaneously examines the disparate signals carried within a set of voxels rather than examining individual voxels in parallel, as is done in the univariate approaches that are used in the vast majority of fMRI studies. By considering multiple responses jointly, MVPA can reveal signal components that are independent of the average regional response. Extending this principle to whole brain fMRI data, MVPA can be used to provide an information-theoretic framework for the isolation of regions that uniquely represent a behavior (Hampton and O'doherty, 2007; Carter et al., 2012). In principle, MVPA also holds the capability to describe brain function at sub-voxel levels (Kriegeskorte et al., 2010), filling the glaring gap between our knowledge of small groups of neurons and the body of research describing functional characteristics of the brain.

While a number of techniques from statistical learning have been applied to fMRI data, here we focus on the use of multivariate pattern classification (MVPC) to decode mental states. Due to the poor generalization of models that utilize whole brain data, most analyses apply some form of feature selection to limit model complexity and improve generalizability. Here we utilize a common MVPC feature selection method which isolates local spheres of voxels and generates a separate model for each sphere, commonly referred to as the searchlight approach (Kriegeskorte et al., 2006). The use of searchlights focuses inference on patterning of information within a given localized area, meeting our goal of bridging voxel-wise information and regional coding.

The classification algorithms used in MVPC can generally be divided into two categories. Linear classification algorithms (Figure 1A, top) use a weighted combination of signals from voxels within a feature set (e.g., a brain region) to decode perceptual or cognitive states. These methods show a measurable benefit in 

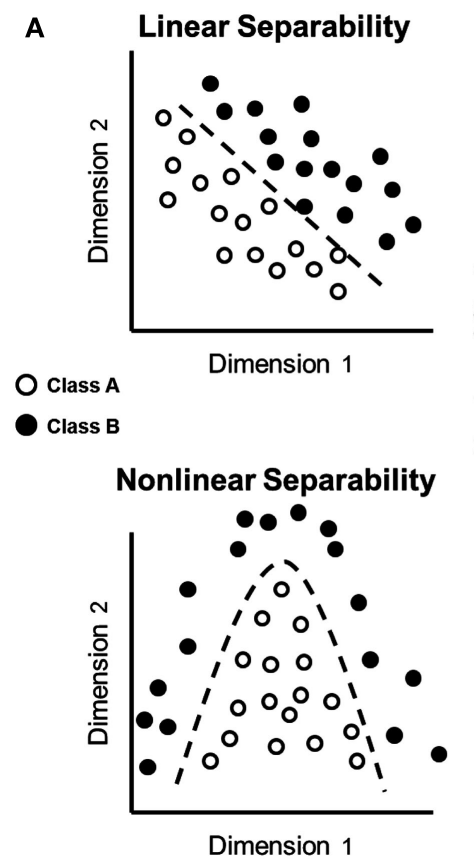

FIGURE 1 | Learning algorithms in multivariate pattern classification (MVPC) of fMRI studies. (A) Pattern classification problems can be identified as linearly separable or inseparable, depending upon how component features encode information. Solutions for linear and non-linear pattern separation are depicted as detailed by Mangasarian (1965). If linearly separable, an $n$-dimensional planar surface defined by the point $x$ weighted by the vector $d$ and offset by the scalar $\gamma$ can successfully separate the patterns $A$ and $B(x d-\gamma=0 \mid A d-e \gamma>0, B d-l \gamma<0)$. In the case of quadratic separability shown here, an additional term can be added creating a non-linear

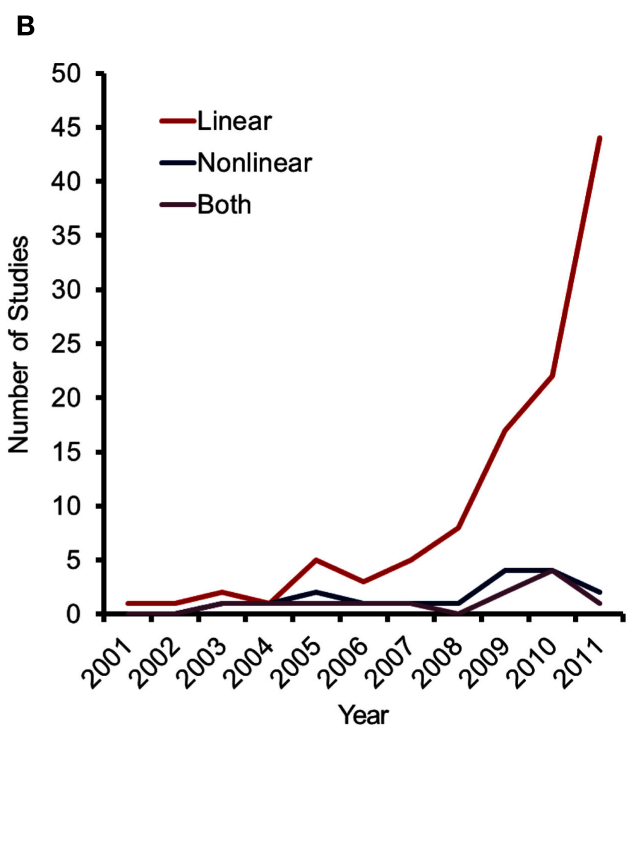

surface that separates $A$ and $B\left(x E x^{\prime}+x d-\gamma=0 \mid A_{i} E A_{i}^{\prime}+A_{i} d-\gamma>0\right.$, $B_{j} E B_{j}{ }^{\prime}+B_{j} d-\gamma<0$ ). (B) Number of publications using linear and non-linear algorithms in our meta-analysis of the neuroscience literature broken down by year, showing recent growth in the use of linear rather than non-linear algorithms. The analysis was accomplished by searching PubMed on August 29, 2011 for the terms (fMRI or MRI) and [MVPA or decoding or (pattern classification)], identifying studies from that search that used pattern classification to study brain function - with the assistance of the AntConc corpus analysis toolkit (Anthony, 2011). signal detection beyond using a univariate general linear model. However, each individual voxel must still contain information that can separate the stimuli of interest (see Mangasarian, 1965 for a mathematical description). In contrast, non-linear classification algorithms (Figure 1A, bottom) use a complex combination of information across voxels (e.g., a polynomial, sinusoid, or Gaussian function) so that even voxels that contain no useful information by themselves may still improve the classification performance of a larger set of voxels. Thus, linear and non-linear classifiers are capable of characterizing different types of neural representations (Rasmussen et al., 2011).

In addition to characterizing the type of information encoded within a brain region, comparing linear and non-linear classifiers may offer insight into how activation may be read out or manipulated through subsequent processing steps. For example, if the activation of a region can be decoded using linear classification, functionally connected regions could make use of the identified differences directly; without additional processing (for further discussion, see Norman et al., 2006; Misaki et al., 2010). Conversely, a significant non-linear classification (in the absence of linear decoding) suggests any information represented in that region will require further processing in order to be utilized (Kouh and Poggio, 2008). An example of the distinction is present in work by Kamitani and Tong (2005), where linear ensemble classifiers are sufficient to decode the orientation of perceived lines from patterns of fMRI activity in early visual cortex. Orientation information has been identified, and is being utilized immediately without extended processing. Using a non-linear classifier could allow decoding of face representations in these same voxels, even though their underlying neuronal activity would not be explicitly coding for those representations (i.e., the integration process would happen at a later stage in processing). In this regard, contrasting the decoding capability of linear and non-linear classification may give insight into whether multivariate information is at an early or late stage of a processing stream.

The capacity of non-linear classification algorithms to decode complex patterns comes with a cost. As the complexity of a classifier is increased relative to the quality and amount of data available (e.g., by increasing the number of features or by using algorithms with more parameters), the possibility of overfitting is more likely (Duda and Hart, 1973). Measures of complexity, such as the Vapnik-Chervonenkis dimension (Vapnik and Chervonenkis, 1971) or Rademacher complexity (Bartlett and Mendelson, 2001), are useful in constructing classifiers because they allow one to assess the feasibility of learning on out of sample data (Blumer et al., 1989). For example, non-linear classifiers may estimate an overly complex decision boundary, resulting in poor performance in tests of generalization. Overfitting a complex function may 
obscure linear relationships that are present in the data, although methods such as regularization and cross-validation have been developed to mitigate this problem (Mitchell, 1997). One such approach is the use of spatial regularization, whereby prior information about the spatial relationships of voxels is used to guide classification (Martinez-Ramon et al., 2006; Meyer and Xilin, 2008; Xiang et al., 2009). While the potential for overfitting is high in the case of fMRI (Misaki et al., 2010; Pereira and Botvinick, 2011), several studies (e.g., Hanson et al., 2004; Rasmussen et al., 2011) have demonstrated that pattern classifiers are capable of decoding information bound in non-linear relationships across multivariate samples. Thus, while overfitting is conventionally considered a drawback it can also serve as a marker for excessive model complexity.

Accordingly, the complexity of decoding algorithms should ideally reflect the way in which information is encoded. For example, the neural coding of motion in the occipital gyrus has been investigated at multiple levels of analysis. Brain structures responsible for encoding visual motion (Zeki et al., 1991; Tootell et al., 1995) have been identified using univariate approaches, while more specific information such as the direction of perceived motion has been successfully decoded using linear pattern classification (Kamitani and Tong, 2006). Indeed, the use of pattern analysis is redefining the limits of fMRI, revealing information encoded at spatial scales thought to be beyond the resolution afforded by current technology (Kamitani and Sawahata, 2010; Kriegeskorte et al., 2010), although the origin of this information is debated (Op De Beeck, 2010a,b; Shmuel et al., 2010; Freeman et al., 2011). These findings demonstrate that the improved sensitivity and use of spatial information by multivariate classifiers permit the decoding of more complex information than standard univariate approaches.

Linear and non-linear classifiers are capable of solving different types of classification problems and therefore can provide differential insight into brain function - yet, in practice they are applied to similar research questions. To characterize the usage of these two classes of decoding methods in the literature, we conducted a meta-analysis of fMRI studies employing MVPA methods. Strikingly, 110 of 115 studies (95.7\%) used linear algorithms while only $16(13.9 \%)$ utilized a non-linear approach and 11 studies implemented both methods. Although interpretability and resilience to overfitting in high dimensional datasets warrants the utilization of linear algorithms, their disproportionate use critically limits the types of information that can be decoded from patterns of neural activity. As MVPC is capable of decoding information at multiple levels of complexity and is being used at a rapidly increasing rate (Figure 1B), understanding what information is extracted by these different methods can guide future research.

\section{ENCODING AND DECODING SIGNALS}

To examine how a focus on linear classifiers limits the scope of MVPC, we created simulated data with different spatial distributions of information and attempted to decode them using standard univariate, linear multivariate, and non-linear multivariate models. The simulations were tailored to demonstrate how different schemes of information encoding are decoded with markedly different performance depending on the classification method applied. For the sake of simplicity, the data contained properties similar to those from a single slice acquisition of blood-oxygenlevel-dependent (BOLD) fMRI. We crafted four datasets where embedded signals differentiated between an alternating sequences of time (referred to as states $A$ and $B$ ), as is common to experimental tasks with a blocked design (Figure 2; MATLAB code for constructing and analyzing the datasets is available online at http://www.duke.edu/ pak5/). Each dataset consisted of a 12-by12 matrix sampled at 240 time points in which consecutive blocks of 10 time points alternate between states $A$ and $B$. Signal discriminating the two states was incorporated within a circle of radius 3 voxels above $10 \mathrm{~dB}$ white Gaussian noise. Finally, a spatial filter (Gaussian kernel with FWHM of 3 voxels) was applied to reflect the inherent smoothness of fMRI data.

In particular, we were interested in the relative sensitivity of each analytical approach to information with particular spatiotemporal properties. In the case of univariate analyses, we compared the mean of each voxel during state $A$ and $B$ using a two tailed paired $t$-test. Classification was performed using linear and radial basis function support vector machines implemented in libSVM (Chang and Lin, 2011). We implemented a searchlight approach (Kriegeskorte et al., 2006) constructing multiple classifiers using data from each sample and its four nearest neighbors. This approach highlights how spatial smoothing inherent in fMRI acquisition may impact detection of information of nearby sources. To produce a generalized estimate of accuracy, twelve-fold leave-one-out cross-validation was performed (i.e., the analyses were repeated twelve times, each time partitioning a different block of data for an independent test). The statistical significance of accuracy estimates were then computed using binomial tests against chance levels of performance. Statistical maps produced from univariate and MVPC analyses were then thresholded at a family wise error corrected level of $p<0.05$. Additionally, to compare the relative sensitivity and specificity of different signal detection methods, the area under the receiver operating characteristic curve was computed for all analyses. The hit rate was calculated as the proportion of significant voxels above threshold within the source of information. The false positive rate was similarly calculated using the same procedure, only on data containing smooth noise - no informative signal was added.

In our first example (univariate encoding), we simulated a dataset whose spatial properties were well-matched to the strengths of univariate statistics. We focused on two key properties in constructing this simulation. First, spatial location is not relevant to the information contained in the signal, so independent random sampling from the circle should yield consistent results. Second, the voxels do not interact and can be considered one at a time without a loss of information. Thus, performing a statistical test on each sample separately should be sufficient to identify where the signal is contained. To meet these requirements, we made each sample within the circle deactivated during state $A$ and activated state $B$, resulting in a square waveform. We found that all statistical approaches correctly identified the majority of voxels within the circle. The enhanced sensitivity of MVPC was evident around the boundary of the activated region, where spatial smoothing decreased the information content of voxels. While the univariate approach only identified voxels within the circle, the pattern classifiers could identify voxels just outside the boundary 

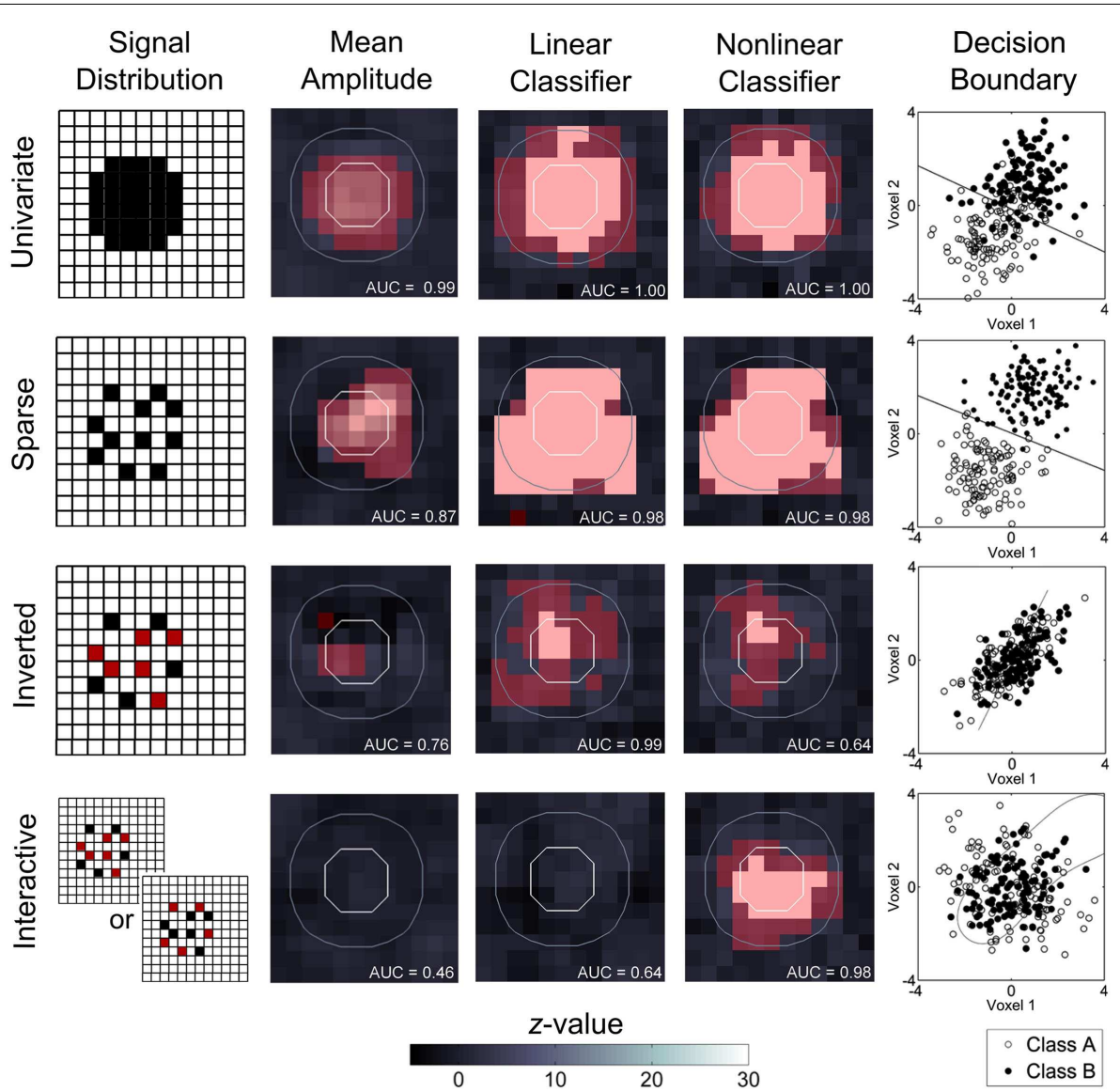

FIGURE 2 |The relative information sensitivity of different fMRI analysis approaches. Simulated datasets comprised a 12-by-12 matrix sampled at 240 time points in which consecutive blocks of 10 time points alternate between two states $(A$ and $B$ ). Signal discriminating the two states is present in a circle of radius 3 voxels above $10 \mathrm{~dB}$ white Gaussian noise. Circles marked with white lines indicate amount of encoded information, with the inner circle containing informative patterns when sampled with a searchlight, while the outer circle may contain some information as a result of spatial smoothing (Gaussian kernel with FWHM of 3 voxels). Red coloring indicates successfully decoded voxels at a family wise error corrected threshold of $p<0.05$. Signal detection is quantified using area under the receiver operating characteristic curve $(\mathrm{AUC})$. In the univariate encoding simulation, information that is encoded by the mean activity of each sample independently, with a homogeneous spatial distribution, is successfully decoded by all methods. For the example with sparse encoding, information present in the mean activity and spatial location of each sample is detected by all three analysis approaches, although MVPC provides increased sensitivity. In the inverted encoding simulation, detection performance is greater for both MVPC approaches than for univariate approaches. And, in the interactive encoding simulation, where embedded signals interact in a state dependent manner to produce information, only the non-linear approach was capable of successfully identifying the embedded signal. where only a small amount of information was present due to the smoothness of the data. Thus, for this simplest case, the advantage of MVPC was that of increased sensitivity compared to univariate techniques.

For our second exemplar dataset (sparse encoding), we simulated a sparsely distributed signal within an activated region by randomly retaining one quarter of the voxels within the circle and increasing their amplitude four fold, ensuring the total amount of signal within the source remained constant. In this case, only some spatial locations provide information that differentiates the two states. For this reason, univariate tests may fail to identify a sample with low or intermediate amplitude, whereas multivariate methods can utilize spatial information to successfully classify the data. Statistical analysis on this dataset revealed that while all three methods could successfully identify that task-related signal was present in the dataset, the univariate analysis failed to identify some regions within the circle.

In the third example (inverted encoding), our goal was to demonstrate a less conventional dependence on spatial location. To accomplish this, we made every sample contain signal but reversed its sign in half the voxels selected at random. By reversing the sign of activity in half of the voxels, both the sign of the signal and its spatial location are required to decode the current state. Consistent with the previous example, multivariate pattern classifiers excelled at decoding spatial information while univariate analysis failed to detect the majority of voxels within the source. Taken together, the results from examples two and three demonstrate the increased sensitivity of MVPC over univariate approaches, with linear and non-linear classifiers exhibiting similar performance. 
For the fourth example (interactive encoding), we created a source in which information is carried exclusively through the interaction of voxels. This was accomplished by randomly distributing two signals throughout the circle in a fashion similar to example three, but allowing the direction of activation to vary over time. In state $A$ the regions alternate between being positive or negative with opposite signs, whereas in state $B$ neither region is active. Classifying the current state requires creating a model that incorporates the interactions between voxels, because the overall activation of any voxel (or the region as a whole) carries no information. As expected, statistical analysis revealed that non-linear classification was the only approach that successfully identified the source of information in the data.

\section{EXPANDING THE USE OF PATTERN CLASSIFICATION}

Consistent with its increasing prevalence in the literature, MVPC has several distinct advantages over conventional univariate approaches. It has greater sensitivity for identifying small effects, especially when the spatial distribution of activity is heterogeneous. This result is consistent with studies finding functional organization in brain structures, such as visual cortex (Kamitani and Tong, 2005) and the hippocampus (Hassabis et al., 2009), that had been previously missed using univariate methods. Studies using linear classifiers to extract unexpected effects have popularized pattern analysis. We found, however, that the benefits of MVPC go well beyond a simple increase in detection power. Linear classifiers provide access to spatial information on top of that carried in the mean level of activity, while non-linear classifiers reveal information likely to be carried in complex computational maps.

The defining properties of linear MVPC are its use of spatial information and focus on individual voxels. Because the distribution of neurons sampled in a voxel can vary with spatial location, spatially sparse fMRI activity is likely a result of heterogeneity in underlying neural populations. While univariate methods gloss over these distinctions by examining each voxel separately, linear classification carries intuitive advantages. As has been commonly noted (Kamitani and Tong, 2005; Norman et al., 2006; Pereira et al., 2009), linear classifiers pool the information contained within individual voxels. This is a useful property when the goal of classification is to leverage fine scale spatial organization in studying brain function because the location of voxels, rather than interactions between them, will drive performance of classification. The combination of these two properties allows the method to reveal information beyond univariate approaches (Jimura and Poldrack, 2012) in a manner that is straightforward to interpret. Thus, linear classification should be employed when neural signals do not interact and are expected to be in a fixed spatial configuration, as in the sparse encoding and inverted encoding simulations.

Non-linear classification, on the other hand, should be harnessed when cognitive states do not necessarily correspond to the

\section{REFERENCES}

Anthony, L. (2011). "AntConc"

(Version 3.2.4). (Tokyo: Waseda University).

Bartlett, P. L., and Mendelson, S. (2001). Rademacher and Gaussian complexities: risk bounds and structural results. J. Mach. Learn. Res. 2111, 224-240.

Blumer, A., Ehrenfeucht, A., Haussler, D., and Warmuth, M. K. (1989). Learnability and the VapnikChervonenkis dimension. J. Altern. Complement. Med. 36, 929-965.

activation of individual voxels, but instead have differential effects depending on the functional properties of those voxels. Because non-linear algorithms treat the activity of a voxel as part of a combinatorial code rather than a unitary piece of information, they are better served to decode more complex representations across association cortex (Hanson et al., 2004) that build upon basic features in primary sensory cortex (e.g., Kamitani and Tong, 2005).This capacity may prove critical in representing multiple different categories from more basic properties in a robust, efficient manner (Op De Beeck et al., 2008). Further, our results demonstrated that non-linear algorithms can identify combinatorial patterns that are not time invariant, but drastically change over time despite containing the same information content. These findings suggest that non-linear classification is an advantageous methodological choice when neural signals are highly intricate and vary over time, for example in interactive encoding.

Despite their benefits and unique capacities, both linear and non-linear classifiers utilize information contained in the activity and spatial location of a sample, which can lead them to show similar results in many cases. The performance of MVPC methods must be compared to univariate results before claims about spatial information can be made. More specifically, a linear classifier must reveal information beyond that which is detectable by univariate methods before the spatial location of inputs can be considered important. Similarly, non-linear classifiers do not only decode pattern activity; mean levels of activity or spatial location can also drive the performance of these learning algorithms. We found comparisons between analysis methods to be infrequent in our meta-analysis, with comparisons to univariate methods being made in 16 (13.9\%) studies and comparisons between non-linear and linear algorithms in only $11(9.6 \%)$ studies. This is especially important since sensitivity in MVPC varies with signal amplitude (Smith et al., 2011). Additionally, given that non-linear classifiers can properly model decision boundaries that linear classifiers are incapable of solving (e.g., the XOR problem or our related interactive encoding example), the application of non-linear classifiers may lead to refinement of models already established with linear approaches. Model comparisons are essential in revealing information contained in non-linear relationships above and beyond the capacity of linear classifiers. While linear classifiers can be successfully applied to improve the sensitivity of fMRI in cases of functionally organized neural activity, they need to be complemented with non-linear algorithms to be a true advance over traditional approaches.

\section{ACKNOWLEDGMENTS}

This research was supported by NIMH R01-86712 and NIMH RC1-88680. Scott A. Huettel was supported by an Incubator Award from the Duke Institute for Brain Sciences.

Boulton, A. A., Baker, G. B., and Vanderwolf, C. H. (1990). Neurophysiological Techniques. Clifton, NJ: Humana Press.

Carter, R. M., Bowling, D. L., Reeck, C., and Huettel, S. A. (2012). A distinct role of the temporal-parietal junction in predicting socially guided decisions. Science 337, 109-111.

Chang, C.-C., and Lin, C.-J. (2011). LIBSVM: A library for support vector machines. ACM Trans. Intell. Syst. Technol. 2, 1-27. 
Dayan, P., and Abbott, L. F. (2001). Theoretical Neuroscience: Computational and Mathematical Modeling of Neural Systems. Cambridge, MA: Massachusetts Institute of Technology Press.

Duda, R. O., and Hart, P. E. (1973). Pattern Classification and Scene Analysis. New York: Wiley.

Fox, M. D., Snyder, A. Z., Vincent, J. L., Corbetta, M., Van Essen, D. C., and Raichle, M. E. (2005). The human brain is intrinsically organized into dynamic, anticorrelated functional networks. Proc. Natl. Acad. Sci. U.S.A. 102, 9673-9678.

Frackowiak, R. S. J. (2004). Human Brain Function. Amsterdam, Boston: Elsevier Academic Press.

Freeman, J., Brouwer, G. J., Heeger, D. J., and Merriam, E. P. (2011). Orientation decoding depends on maps, not columns. J. Neurosci. 31, 4792-4804.

Gerstner, W., and Kistler, W. M. (2002). Spiking Neuron Models: Single Neurons, Populations, Plasticity. Cambridge, NY: Cambridge University Press.

Hampton, A. N., and O'doherty, J. P. (2007). Decoding the neural substrates of reward-related decision making with functional MRI. Proc. Natl. Acad. Sci. U.S.A. 104, 1377-1382.

Hanson, S. J., Matsuka, T., and Haxby, J. V. (2004). Combinatorial codes in ventral temporal lobe for object recognition: Haxby (2001) revisited: is there a "face" area? Neuroimage 23, 156-166.

Hassabis, D., Chu, C., Rees, G., Weiskopf, N., Molyneux, P. D., and Maguire, E. A. (2009). Decoding neuronal ensembles in the human hippocampus. Curr. Biol. 19, 546-554.

Haynes, J. D., and Rees, G. (2006). Decoding mental states from brain activity in humans. Nat. Rev. Neurosci. 7, 523-534.

Huettel, S. A., Song, A. W., and Mccarthy, G. (2008). Functional Magnetic Resonance Imaging. Sunderland, MA: Sinauer Associates.

Jimura, K., and Poldrack, R. A. (2012). Analyses of regional-average activation and multivoxel pattern information tell complementary stories. Neuropsychologia 50, 544-552.

Kamitani, Y., and Sawahata, Y. (2010). Spatial smoothing hurts localization but not information: pitfalls for brain mappers. Neuroimage 49, 1949-1952.

Kamitani, Y., and Tong, F. (2005). Decoding the visual and subjective contents of the human brain. Nat. Neurosci. 8, 679-685.

Kamitani, Y., and Tong, F. (2006). Decoding seen and attended motion directions from activity in the human visual cortex. Curr. Biol. 16, 1096-1102.

Kim, D. S., Duong, T. Q., and Kim, S. G. (2000). High-resolution mapping of iso-orientation columns by fMRI. Nat. Neurosci. 3, 164-169.

Koch, C. (1997). Computation and the single neuron. Nature 385, 207-210.

Kouh, M., and Poggio, T. (2008). A canonical neural circuit for cortical nonlinear operations. Neural. Comput. 20, 1427-1451.

Kriegeskorte, N., and Bandettini, P. (2007). Analyzing for information, not activation, to exploit highresolution fMRI. Neuroimage 38, 649-662.

Kriegeskorte, N., Cusack, R., and Bandettini, P. (2010). How does an fMRI voxel sample the neuronal activity pattern: compact-kernel or complex spatiotemporal filter? Neuroimage 49, 1965-1976.

Kriegeskorte, N., Goebel, R., and Bandettini, P. (2006). Informationbased functional brain mapping. Proc. Natl. Acad. Sci. U.S.A. 103, 3863-3868.

Kriegeskorte, N., Mur, M., and Bandettini, P. (2008). Representational similarity analysis - connecting the branches of systems neuroscience. Front. Syst. Neurosci. 2:4. doi:10.3389/neuro.06.004.2008

Logothetis, N. K., Pauls, J., Augath, M., Trinath, T., and Oeltermann, A. (2001). Neurophysiological investigation of the basis of the fMRI signal. Nature 412, 150-157.

Mangasarian, O. L. (1965). Linear and nonlinear separation of patterns by linear programming. Oper. Res. 13, 444-452.

Martinez-Ramon, M., Koltchinskii, V., Heileman, G. L., and Posse, S. (2006). fMRI pattern classification using neuroanatomically constrained boosting. Neuroimage 31, 1129-1141.

Meyer, F. G., and Xilin, S. (2008). Classification of fMRI time series in a lowdimensional subspace with a spatial prior. IEEE Trans. Med. Imaging 27, 87-98.

Misaki, M., Kim, Y., Bandettini, P. A., and Kriegeskorte, N. (2010). Comparison of multivariate classifiers and response normalizations for pattern-information fMRI. $\mathrm{Neu}$ roimage 53, 103-118.

Mitchell, T. M. (1997). Machine Learning. New York: McGraw-Hill.

Mountcastle, V. B. (1997). The columnar organization of the neocortex. Brain 120(Pt 4), 701-722.

Norman, K. A., Polyn, S. M., Detre, G. J., and Haxby, J. V. (2006). Beyond mind-reading: multi-voxel pattern analysis of fMRI data. Trends Cogn. Sci. (Regul. Ed.) 10, 424-430.

Op De Beeck, H. P. (2010a). Against hyperacuity in brain reading: spatial smoothing does not hurt multivariate fMRI analyses? Neuroimage 49, 1943-1948.

Op De Beeck, H. P. (2010b). Probing the mysterious underpinnings of multivoxel fMRI analyses. Neuroimage 50, 567-571.

Op De Beeck, H. P., Haushofer, J., and Kanwisher, N. G. (2008). Interpreting fMRI data: maps, modules and dimensions. Nat. Rev. Neurosci. 9, 123-135.

Pereira, F., and Botvinick, M. (2011). Information mapping with pattern classifiers: a comparative study. $\mathrm{Neu}$ roimage 56, 476-496.

Pereira, F., Mitchell, T., and Botvinick, M. (2009). Machine learning classifiers and fMRI: a tutorial overview. Neuroimage 45, S199-S209.

Rasmussen, P. M., Madsen, K. H., Lund, T. E., and Hansen, L. K. (2011). Visualization of nonlinear kernel models in neuroimaging by sensitivity maps. Neuroimage 55, 1120-1131.

Rolls, E. T., and Treves, A. (1998). Neural Networks And Brain Function. New York: Oxford University Press.

Shmuel, A., Chaimow, D., Raddatz, G. Ugurbil, K., and Yacoub, E. (2010). Mechanisms underlying decoding at $7 \mathrm{~T}$ : ocular dominance columns, broad structures, and macroscopic blood vessels in V1 convey information on the stimulated eye. Neuroimage 49, 1957-1964.

Smith, A. T., Kosillo, P., and Williams, A. L. (2011). The confounding effect of response amplitude on MVPA performance measures. Neuroimage 56 525-530.
Tootell, R. B., Reppas, J. B., Kwong, K. K., Malach, R., Born, R. T., Brady, T. J., et al. (1995). Functional analysis of human MT and related visual cortical areas using magnetic resonance imaging. J. Neurosci. 15 , 3215-3230.

Vapnik, V. N., and Chervonenkis, A. Y. A. (1971). Theory of uniform convergence of frequencies of events to their probabilities and problems of search for an optimal solution from empirical data. IEEE Trans. Automat. Contr. 32, 207.

Weil, R. S., and Rees, G. (2010). Decoding the neural correlates of consciousness. Curr. Opin. Neurol. 23, 649-655.

Xiang, Z., Xi, Y., Hasson, U., and Ramadge, P. (2009). "Boosting with spatial regularization," in Advances in Neural Information Processing Systems, eds Y. Bengio, D. Schuurmans J. Lafferty, C. K. I. Williams and A. Culotta Vol. 22 (Vancouver), 21072115.

Zeki, S., Watson, J. D., Lueck, C. J., Friston, K. J., Kennard, C., and Frackowiak, R. S. (1991). A direct demonstration of functional specialization in human visual cortex. J. Neurosci. 11, 641-649.

Conflict of Interest Statement: The authors declare that the research was conducted in the absence of any commercial or financial relationships that could be construed as a potential conflict of interest.

Received: 02 July 2012; accepted: 22 October 2012; published online: 23 November 2012.

Citation: Kragel PA, Carter RM and Huettel SA (2012) What makes a pattern? Matching decoding methods to data in multivariate pattern analysis. Front. Neurosci. 6:162. doi: 10.3389/fnins.2012.00162

This article was submitted to Frontiers in Brain Imaging Methods, a specialty of Frontiers in Neuroscience.

Copyright (c) 2012 Kragel, Carter and Huettel. This is an open-access article distributed under the terms of the Creative Commons Attribution License, which permits use, distribution and reproduction in other forums, provided the original authors and source are credited and subject to any copyright notices concerning any third-party graphics etc. 


\section{APPENDIX \\ MANUSCRIPTS INCLUDED IN META-ANALYSIS}

Abrams, D. A., Bhatara, A., Ryali, S., Balaban, E., Levitin, D. J., and Menon, V. (2011). Decoding temporal structure in music and speech relies on shared brain resources but elicits different fine-scale spatial patterns. Cereb. Cortex 21, 1507-1518.

Alink, A., Euler, F., Kriegeskorte, N., Singer, W., and Kohler, A. (2011). Auditory motion direction encoding in auditory cortex and high-level visual cortex. Hum. Brain Mapp. 33, 969-978.

Bach, D. R., Weiskopf, N., and Dolan, R. J. (2011). A stable sparse fear memory trace in human amygdala. J. Neurosci. 31, 9383-9389.

Baucom, L. B., Wedell, D. H., Wang, J., Blitzer, D. N., and Shinkareva, S. V. (2011). Decoding the neural representation of affective states. $\mathrm{Neu}$ roimage 59, 718-727.

Beauchamp, M. S., Laconte, S., and Yasar, N. (2009). Distributed representation of single touches in somatosensory and visual cortex. Hum. Brain Mapp. 30, 3163-3171.

Bode, S., and Haynes, J. D. (2009). Decoding sequential stages of task preparation in the human brain. Neuroimage 45, 606-613.

Bode, S., He, A. H., Soon, C. S., Trampel, R., Turner, R., and Haynes, J. D. (2011). Tracking the unconscious generation of free decisions using uitra-high field fMRI. PLoS ONE 6, e21612. doi:10.1371/journal.pone.0021612

Bonnici, H. M., Kumaran, D., Chadwick, M. J., Weiskopf, N., Hassabis, D., and Maguire, E. A. (2011). Decoding representations of scenes in the medial temporal lobes. Hippocampus 22, 1143-1153.

Brouwer, G. J., and Heeger, D. J. (2009). Decoding and reconstructing color from responses in human visual cortex. J. Neurosci. 29, 13992-14003.

Carlson, T., Hogendoorn, H., Fonteijn, H., and Verstraten, F. A. (2011). Spatial coding and invariance in objectselective cortex. Cortex 47, 14-22.

Carlson, T. A., Schrater, P., and He, S. (2003). Patterns of activity in the categorical representations of objects. J. Cogn. Neurosci. 15, 704-717.

Chadwick, M. J., Hassabis, D., Weiskopf, N., and Maguire, E. A. (2010). Decoding individual episodic memory traces in the human hippocampus. Curr. Biol. 20, 544-547.

Chaimow, D., Yacoub, E., Ugurbil, K., and Shmuel, A. (2011). Modeling and analysis of mechanisms underlying fMRI-based decoding of information conveyed in cortical columns. Neuroimage 56, 627-642.

Chang, K. M., Mitchell, T., and Just, M. A. (2011). Quantitative modeling of the neural representation of objects: how semantic feature norms can account for fMRI activation. Neuroimage 56, 716-727.

Cichy, R. M., Chen, Y., and Haynes, J. D. (2011a). Encoding the identity and location of objects in human LOC. Neuroimage 54, 2297-2307.

Cichy, R. M., Heinzle, J., and Haynes, J. D. (2011b). Imagery and perception share cortical representations of content and location. Cereb. Cortex.

Clithero, J. A., Carter, R. M., and Huettel, S. A. (2009). Local pattern classification differentiates processes of economic valuation. Neuroimage 45, 1329-1338

Clithero, J. A., Smith, D. V., Carter, R. M., and Huettel, S. A. (2011). Within- and cross-participant classifiers reveal different neural coding of information. Neuroimage 56, 699-708.

Costafreda, S. G., Fu, C. H., Picchioni, M., Toulopoulou, T., Mcdonald, C., Kravariti, E., et al. (2011). Pattern of neural responses to verbal fluency shows diagnostic specificity for schizophrenia and bipolar disorder. BMC Psychiatry 11, 18. doi:10.1186/1471-244X-11-18

Coutanche, M. N., Thompson-Schill, S. L., and Schultz, R. T. (2011). Multivoxel pattern analysis of fMRI data predicts clinical symptom severity. Neuroimage 57, 113-123.

Cox, D. D., and Savoy, R. L. (2003). Functional magnetic resonance imaging (fMRI) "brain reading": detecting and classifying distributed patterns of fMRI activity in human visual cortex. Neuroimage 19, 261-270.

Craddock, R. C., Holtzheimer, P. E. III., Hu, X. P., and Mayberg, H. S. (2009). Disease state prediction from resting state functional connectivity. Magn. Reson. Med. 62, 1619-1628.

Davatzikos, C., Ruparel, K., Fan, Y., Shen, D. G., Acharyya, M., Loughead, J. W., et al. (2005). Classifying spatial patterns of brain activity with machine learning methods: application to lie detection. Neuroimage 28, 663-668.

Dinstein, I., Gardner, J. L., Jazayeri, M., and Heeger, D. J. (2008). Executed and observed movements have different distributed representations in human aIPS. J. Neurosci. 28, 11231-11239.
Eger, E., Ashburner, J., Haynes, J. D., Dolan, R. J., and Rees, G. (2008). fMRI activity patterns in human LOC carry information about object exemplars within category. J. Cogn Neurosci. 20, 356-370.

Esterman, M., Chiu, Y. C., TamberRosenau, B. J., and Yantis, S. (2009). Decoding cognitive control in human parietal cortex. Proc. Natl. Acad. Sci. U.S.A. 106, 17974-17979.

Ethofer, T., Van De Ville, D., Scherer, K. and Vuilleumier, P. (2009). Decoding of emotional information in voice-sensitive cortices. Curr. Biol. 19, 1028-1033.

Etzel, J. A., Gazzola, V., and Keysers, C. (2008). Testing simulation theory with cross-modal multivariate classification of fMRI data. PLoS ONE 3, e3690. doi:10.1371/journal.pone.0003690

Formisano, E., De Martino, F., Bonte, M., and Goebel, R. (2008). "Who" is saying "what"? Brain-based decoding of human voice and speech. Science 322, 970-973.

Freeman, J., Brouwer, G. J., Heeger, D. J., and Merriam, E. P. (2011). Orientation decoding depends on maps, not columns. J. Neurosci. 31, 4792-4804.

Fu, C. H., Mourao-Miranda, J., Costafreda, S. G., Khanna, A., Marquand, A. F., Williams, S. C., and Brammer, M. J. (2008). Pattern classification of sad facial processing: toward the development of neurobiological markers in depression. Biol. Psychiatry 63, 656-662.

Gallivan, J. P., Mclean, D. A., Valyear, K. F., Pettypiece, C. E., and Culham, J. C. (2011). Decoding action intentions from preparatory brain activity in human parieto-frontal networks. J. Neurosci. 31, 9599-9610.

Gilbert, S. J. (2011). Decoding the content of delayed intentions. $\mathrm{J}$. Neurosci. 31, 2888-2894.

Gilbert, S. J., Meuwese, J. D., Towgood, K. J., Frith, C. D., and Burgess, P. W. (2009). Abnormal functional specialization within medial prefrontal cortex in high-functioning autism: a multi-voxel similarity analysis. Brain 132, 869-878.

Greenberg, A. S., Esterman, M., Wilson, D., Serences, J. T., and Yantis, S. (2010). Control of spatial and feature-based attention in frontoparietal cortex. J. Neurosci. 30, 14330-14339.

Hahn, T., Marquand, A. F., Ehlis, A. C., Dresler, T., Kittel-Schneider, S., Jarczok, T. A., et al. (2011). Integrating neurobiological markers of depression. Arch. Gen. Psychiatry 68, 361-368.

Hampton, A. N., and O'doherty J, P. (2007). Decoding the neural substrates of reward-related decision making with functional MRI. Proc. Natl. Acad. Sci. U.S.A. 104, 1377-1382.

Hanson, S. J., Matsuka, T., and Haxby, J. V. (2004). Combinatorial codes in ventral temporal lobe for object recognition: Haxby (2001) revisited: is there a "face" area? Neuroimage 23, 156-166.

Harrison, S. A., and Tong, F. (2009). Decoding reveals the contents of visual working memory in early visual areas. Nature 458, 632-635.

Hassabis, D., Chu, C., Rees, G., Weiskopf, N., Molyneux, P. D., and Maguire, E. A. (2009). Decoding neuronal ensembles in the human hippocampus. Curr. Biol. 19, 546-554.

Haxby, J. V., Gobbini, M. I., Furey, M. L., Ishai, A., Schouten, J. L., and Pietrini, P. (2001). Distributed and overlapping representations of faces and objects in ventral temporal cortex. Science 293, 2425-2430.

Haynes, J. D., Sakai, K., Rees, G., Gilbert, S., Frith, C., and Passingham, R. E. (2007). Reading hidden intentions in the human brain. Curr. Biol. 17, 323-328.

Herrmann, B., Obleser, J., Kalberlah, C., Haynes, J. D., and Friederici, A. D. (2011). Dissociable neural imprints of perception and grammar in auditory functional imaging. Hum. Brain Mapp. 33, 584-595.

Hoeft, F., Mccandliss, B. D., Black, J. M., Gantman, A., Zakerani, N., Hulme, C., et al. (2011). Neural systems predicting long-term outcome in dyslexia. Proc. Natl. Acad. Sci. U.S.A. 108, 361-366.

Jehee, J. F., Brady, D. K., and Tong, F. (2011). Attention improves encoding of task-relevant features in the human visual cortex. J. Neurosci. 31, 8210-8219.

Johnson, J. D., Mcduff, S. G., Rugg, M. D., and Norman, K. A. (2009). Recollection, familiarity, and cortical reinstatement: a multivoxel pattern analysis. Neuron 63, 697-708.

Kamitani, Y., and Tong, F. (2005). Decoding the visual and subjective contents of the human brain. Nat. Neurosci. 8, 679-685.

Kamitani, Y., and Tong, F. (2006). Decoding seen and attended motion directions from activity in the human visual cortex. Curr. Biol. 16, 1096-1102. 
Kahnt, T., Heinzle, J., Park, S. Q., and Haynes, J. D. (2010). The neural code of reward anticipation in human orbitofrontal cortex. Proc. Natl. Acad. Sci. U.S.A. 107, 6010-6015.

Kahnt, T., Heinzle, J., Park, S. Q., and Haynes, J. D. (2011). Decoding different roles for vmPFC and dIPFC in multi-attribute decision making. Neuroimage 56, 709-715.

Kaul, C., Rees, G., and Ishai, A. (2011). The gender of face stimuli is represented in multiple regions in the human brain. Front. Hum. Neurosci. 4:238. doi:10.3389/fnhum.2010.00238

Kay, K. N., Naselaris, T., Prenger, R. J., and Gallant, J. L. (2008). Identifying natural images from human brain activity. Nature 452, 352-355.

Krajbich, I., Camerer, C., Ledyard, J., and Rangel, A. (2009). Using neural measures of economic value to solve the public goods free-rider problem. Science 326, 596-599.

Kuhn, S., Bodammer, N. C., and Brass, M. (2010). Dissociating mental states related to doing nothing by means of fMRI pattern classification. Neuroimage 53, 1294-1300.

Langleben, D. D., Loughead, J. W., Bilker, W. B., Ruparel, K., Childress, A. R., Busch, S. I., et al. (2005). Telling truth from lie in individual subjects with fast eventrelated fMRI. Hum. Brain Mapp. 26, 262-272.

Lee, Y. S., Janata, P., Frost, C., Hanke, M., and Granger, R. (2011). Investigation of melodic contour processing in the brain using multivariate pattern-based fMRI. Neuroimage 57, 293-300.

Lewis-Peacock, J. A., and Postle, B. R. (2008). Temporary activation of long-term memory supports working memory. J. Neurosci. 28, 8765-8771.

Li, L., Qin, W., Bai, L., and Tian, J. (2010). Exploring vision-related acupuncture point specificity with multivoxel pattern analysis. Magn. Reson. Imaging 28, 380-387.

Li, S., Ostwald, D., Giese, M., and Kourtzi, Z. (2007). Flexible coding for categorical decisions in the human brain. J. Neurosci. 27, 12321-12330.

Li, Y., Wang, G., Long, J., Yu, Z., Huang, B., Li, X., et al. (2011). Reproducibility and discriminability of brain patterns of semantic categories enhanced by congruent audiovisual stimuli. PLoS ONE 6, e20801. doi:10.1371/journal.pone.0020801
Macevoy, S. P., and Epstein, R. A. (2009). Decoding the representation of multiple simultaneous objects in human occipitotemporal cortex. Curr. Biol. 19, 943-947.

Mahon, B. Z., and Caramazza, A. (2010). Judging semantic similarity: an event-related fMRI study with auditory word stimuli. Neuroscience $169,279-286$.

Manelis, A., Hanson, C., and Hanson, S. J. (2011a). Implicit memory for object locations depends on reactivation of encoding-related brain regions. Hum. Brain Mapp. 32, 32-50.

Manelis, A., Reder, L. M., and Hanson, S. J. (2011b). Dynamic changes in the medial temporal lobe during incidental learning of object-location associations. Cereb. Cortex 22, 828-837.

Marquand, A., Howard, M., Brammer, M., Chu, C., Coen, S., and MouraoMiranda, J. (2010). Quantitative prediction of subjective pain intensity from whole-brain fMRI data using Gaussian processes. Neuroimage 49, 2178-2189.

Marquand, A. F., De Simoni, S., O'daly, O. G., Williams, S. C., Mourao-Miranda, J., and Mehta, M. A. (2011). Pattern classification of working memory networks reveals differential effects of methylphenidate, atomoxetine, and placebo in healthy volunteers. Neuropsychopharmacology 36, 1237-1247.

Mcduff, S. G., Frankel, H. C., and Norman, K. A. (2009). Multivoxel pattern analysis reveals increased memory targeting and reduced use of retrieved details during singleagenda source monitoring. J. Neurosci. 29, 508-516.

Natu, V., Raboy, D., and O'toole, A. J. (2011). Neural correlates of ownand other-race face perception: spatial and temporal response differences. Neuroimage 54, 2547-2555.

Nestor, A., Plaut, D. C., and Behrmann, M. (2011). Unraveling the distributed neural code of facial identity through spatiotemporal pattern analysis. Proc. Natl. Acad. Sci. U.S.A. 108, 9998-10003.

Nichols, D. F., Betts, L. R., and Wilson, H. R. (2010). Decoding of faces and face components in face-sensitive human visual cortex. Front. Psychol. 1:28. doi:10.3389/fpsyg.2010.00028

O'toole, A. J., Jiang, F., Abdi, H., and Haxby, J. V. (2005). Partially distributed representations of objects and faces in ventral temporal cortex. $J$. Cogn. Neurosci. 17, 580-590.
Ogawa, K., and Inui, T. (2011). Neural representation of observed actions in the parietal and premotor cortex. Neuroimage 56, 728-735.

Oosterhof, N. N., Wiggett, A. J., Diedrichsen, J., Tipper, S. P., and Downing, P. E. (2010). Surfacebased information mapping reveals crossmodal vision-action representations in human parietal and occipitotemporal cortex. J. Neurophysiol. 104, 1077-1089.

Oztekin, I., and Badre, D. (2011) Distributed patterns of brain activity that lead to forgetting. Front. Hum. Neurosci. 5:86 doi:10.3389/fnhum.2011.00086

Park, S., Brady, T. F., Greene, M. R., and Oliva, A. (2011). Disentangling scene content from spatial boundary: complementary roles for the parahippocampal place area and lateral occipital complex in representing real-world scenes. J. Neurosci. 31, 1333-1340.

Parkes, L. M., Marsman, J. B., Oxley, D. C., Goulermas, J. Y., and Wuerger, S. M. (2009). Multivoxel fMRI analysis of color tuning in human primary visual cortex. J. Vis. 9, 1 1-13.

Peelen, M. V., Atkinson, A. P., and Vuilleumier, P. (2010). Supramodal representations of perceived emotions in the human brain. J. Neurosci. 30, 10127-10134.

Pessoa, L., and Padmala, S. (2007). Decoding near-threshold perception of fear from distributed single-trial brain activation. Cereb. Cortex 17, 691-701.

Poldrack, R. A., Halchenko, Y. O., and Hanson, S. J. (2009). Decoding the large-scale structure of brain function by classifying mental States across individuals. Psychol. Sci. 20, 1364-1372.

Polyn, S. M., Natu, V. S., Cohen, J. D., and Norman, K. A. (2005). Category-specific cortical activity precedes retrieval during memory search. Science 310, 1963-1966.

Ponseti, J., Granert, O., Jansen, O., Wolff, S., Mehdorn, H., Bosinski, H., et al. (2009). Assessment of sexual orientation using the hemodynamic brain response to visual sexual stimuli. $J$. Sex. Med. 6, 1628-1634.

Preston, T. J., Li, S., Kourtzi, Z., and Welchman, A. E. (2008). Multivoxel pattern selectivity for perceptually relevant binocular disparities in the human brain. J. Neurosci. 28, 11315-11327.

Reddy, L., Tsuchiya, N., and Serre, T. (2010). Reading the mind's eye: decoding category information during mental imagery. Neuroimage 50 818-825.
Reverberi, C., Gorgen, K., and Haynes, J. D. (2011). Compositionality of rule representations in human prefrontal cortex. Cereb. Cortex 22, 1237-1246.

Rissman, J., Greely, H. T., and Wagner, A. D. (2010). Detecting individual memories through the neural decoding of memory states and past experience. Proc. Natl. Acad. Sci. U.S.A. 107, 9849-9854.

Rodriguez, P. F. (2010). Neural decoding of goal locations in spatial navigation in humans with fMRI. Hum. Brain Mapp. 31, 391-397.

Rogalsky, C., Rong, F., Saberi, K., and Hickok, G. (2011). Functional anatomy of language and music perception: temporal and structural factors investigated using functional magnetic resonance imaging. J. Neurosci. 31, 3843-3852.

Rolls, E. T., Grabenhorst, F., and Franco, L. (2009). Prediction of subjective affective state from brain activations. J. Neurophysiol. 101, 1294-1308.

Russ, M. O., Cleff, U., Lanfermann, H., Schalnus, R., Enzensberger, W., and Kleinschmidt, A. (2002). Functional magnetic resonance imaging in acute unilateral optic neuritis. $J$. Neuroimaging 12, 339-350.

Said, C. P., Moore, C. D., Engell, A. D., Todorov, A., and Haxby, J. V. (2010). Distributed representations of dynamic facial expressions in the superior temporal sulcus. J. Vis. 10, 11

Sato, J. R., De Oliveira-Souza, R., Thomaz, C. E., Basilio, R., Bramati, I. E., Amaro, E. Jr., et al. (2011). Identification of psychopathic individuals using pattern classification of MRI images. Soc. Neurosci. 1-13.

Schwarzkopf, D. S., Schindler, A., and Rees, G. (2010). Knowing with which eye we see: utrocular discrimination and eye-specific signals in human visual cortex. PLoS ONE 5, e13775. doi:10.1371/journal.pone.0013775

Schwarzkopf, D. S., Sterzer, P., and Rees, G. (2011). Decoding of coherent but not incoherent motion signals in early dorsal visual cortex. Neuroimage 56, 688-698.

Serences, J. T., and Boynton, G. M. (2007). Feature-based attentional modulations in the absence of direct visual stimulation. Neuron 55 , 301-312.

Seymour, K., Clifford, C. W., Logothetis, N. K., and Bartels, A. (2010). Coding and binding of color and form in visual cortex. Cereb. Cortex 20, 1946-1954.

Shen, L., Qi, Y., Kim, S., Nho, K., Wan, J., Risacher, S. L., et al. 
(2010). Sparse bayesian learning for identifying imaging biomarkers in $\mathrm{AD}$ prediction. Med. Image Comput. Comput. Assist. Interv. 13, 611-618.

Shmuel, A., Chaimow, D., Raddatz, G., Ugurbil, K., and Yacoub, E. (2010). Mechanisms underlying decoding at $7 \mathrm{~T}$ : ocular dominance columns, broad structures, and macroscopic blood vessels in V1 convey information on the stimulated eye. Neuroimage 49, 1957-1964.

Sitaram, R., Lee, S., Ruiz, S., Rana, M., Veit, R., and Birbaumer, N. (2011). Real-time support vector classification and feedback of multiple emotional brain states. Neuroimage 56, 753-765.

Smith, F. W., and Muckli, L. (2010). Non-stimulated early visual areas carry information about surrounding context. Proc. Natl. Acad. Sci. U.S.A. 107, 20099-20103.

Tamber-Rosenau, B. J., Esterman, M., Chiu, Y. C., and Yantis, S. (2011). Cortical mechanisms of cognitive control for shifting attention in vision and working memory. J. Cogn. Neurosci. 23, 2905-2919.
Thirion, B., Duchesnay, E., Hubbard, E., Dubois, J., Poline, J. B., Lebihan, D., et al. (2006). Inverse retinotopy: inferring the visual content of images from brain activation patterns. Neuroimage 33, 1104-1116.

Thompson, J. C., and Baccus, W. (2011). Form and motion make independent contributions to the response to biological motion in occipitotemporal cortex. Neuroimage 59, 625-634.

Tusche, A., Bode, S., and Haynes, J. D. (2010). Neural responses to unattended products predict later consumer choices. J. Neurosci. 30, 8024-8031.

Visser, R. M., Scholte, H. S., and Kindt, M. (2011). Associative learning increases trial-by-trial similarity of BOLD-MRI patterns. J. Neurosci. 31, 12021-12028.

Walther, D. B., Caddigan, E., Fei-Fei, L., and Beck, D. M. (2009). Natural scene categories revealed in distributed patterns of activity in the human brain. J. Neurosci. 29, 10573-10581.

Walther, D. B., Chai, B., Caddigan, E., Beck, D. M., and Fei-Fei,
L. (2011). Simple line drawings suffice for functional MRI decoding of natural scene categories. Proc. Natl. Acad. Sci. U.S.A. 108, 9661-9666.

Wang, K., Jiang, T., Liang, M., Wang, L., Tian, L., Zhang, X., et al. (2006). Discriminative analysis of early Alzheimer's disease based on two intrinsically anti-correlated networks with resting-state fMRI. Med. Image Comput. Comput. Assist. Interv. 9, 340-347.

Watanabe, T., Hirose, S., Wada, H., Katsura, M., Chikazoe, J., Jimura, K., et al. (2011). Prediction of subsequent recognition performance using brain activity in the medial temporal lobe. Neuroimage 54 3085-3092.

Williams, A. L., and Smith, A. T. (2010). Representation of eye position in the human parietal cortex. J. Neurophysiol. 104, 2169-2177.

Wolbers, T., Zahorik, P., and Giudice, N. A. (2011). Decoding the direction of auditory motion in blind humans. Neuroimage 56, 681-687.

Woolgar, A., Thompson, R., Bor, D., and Duncan, J. (2011).
Multi-voxel coding of stimuli, rules, and responses in human frontoparietal cortex. Neuroimage 56, 744-752.

Yoon, J. H., Tamir, D., Minzenberg, M. J., Ragland, J. D., Ursu, S., and Carter, C. S. (2008). Multivariate pattern analysis of functional magnetic resonance imaging data reveals deficits in distributed representations in schizophrenia. Biol. Psychiatry 64, 1035-1041.

Zhang, J., Meeson, A., Welchman, A. E., and Kourtzi, Z. (2010). Learning alters the tuning of functional magnetic resonance imaging patterns for visual forms. J. Neurosci. 30, 14127-14133.

Zhang, Y., Tian, J., Yuan, K., Liu, P., Zhuo, L., Qin, W., et al. (2011). Distinct resting-state brain activities in heroin-dependent individuals. Brain Res. 1402, 46-53.

Zhu, C. Z., Zang, Y. F., Liang, M., Tian, L. X., He, Y., Li, X. B., et al. (2005). Discriminative analysis of brain function at resting-state for attention-deficit/hyperactivity disorder. Med. Image Comput. Comput. Assist. Interv. 8, 468-475. 